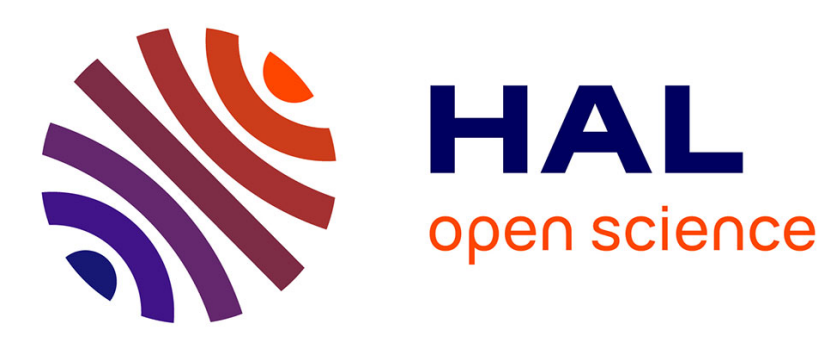

\title{
The 3-PPPS parallel robot with U-shape Base, a 6-DOF parallel robot with simple kinematics
}

Damien Chablat, Luc Baron, Ranjan Jha, Luc Rolland

\section{To cite this version:}

Damien Chablat, Luc Baron, Ranjan Jha, Luc Rolland. The 3-PPPS parallel robot with U-shape Base, a 6-DOF parallel robot with simple kinematics. Advances in Robot Kinematics 2018, 2018, 978-3-319-93187-6. 10.1007/978-3-319-93188-3_23 . hal-01782286

\section{HAL Id: hal-01782286 \\ https://hal.science/hal-01782286}

Submitted on 1 May 2018

HAL is a multi-disciplinary open access archive for the deposit and dissemination of scientific research documents, whether they are published or not. The documents may come from teaching and research institutions in France or abroad, or from public or private research centers.
L'archive ouverte pluridisciplinaire HAL, est destinée au dépôt et à la diffusion de documents scientifiques de niveau recherche, publiés ou non, émanant des établissements d'enseignement et de recherche français ou étrangers, des laboratoires publics ou privés. 


\title{
The 3-PPPS parallel robot with U-shape Base, a 6-DOF parallel robot with simple kinematics
}

\author{
Damien Chablat ${ }^{1}$, Luc Baron ${ }^{2}$, Ranjan Jha ${ }^{3}$, and Luc Rolland ${ }^{4}$ \\ 1 CNRS, LS2N, UMR 6004, 1 rue de la Noë, 44321 Nantes, France, \\ Damien.Chablat@cnrs.fr \\ 2 Mechanical Engineering Department, École Polytéchnique de Montréal, H3C 3A7 \\ Québec, Canada \\ Luc.Baron@polymtl.ca \\ 3 Biomedical Instrumentation Unit, CSIR-Central Scientific Instruments \\ Organisation, India \\ Ranjan. Jha@polymtl.ca \\ 4 School of Engineering and Computing, University of the West of Scotland, Paisley, \\ Scotland, UK \\ Luc.Rolland@uws . ac.uk
}

\begin{abstract}
One of the main problems associated with the use of 6 DOF parallel robots remains the solving of their kinematic models. This is rarely possible to analytically solve their models thereby justifying the application of numerical methods. These methods are difficult to implement in an industrial controller and can cause solution bifurcations close to singularities resulting in following an unplanned trajectory. Recently, a 3-PPPS robot with U-shaped base was introduced where an analytical kinematic model can be derived. Previously, quaternion parameters were used to represent the orientation of the mobile platform. To allow for simpler model handling, this article introduces the use of Euler angles which have a physical meaning for the users. Compact writing of the direct and inverse kinematic model is thus obtained. Using algebraic and cylindrical decomposition for the workspace, this provides a simpler representation of the largest domain without singularity around the "home" configuration.
\end{abstract}

Keywords: 6 DOF Parallel robot, Singularity, Kinematic model

\section{Introduction}

The first all-electric robot, namely the Stanford Arm, was created in 1969 by Victor David Scheinman in the Stanford Artificial Intelligence Laboratory (SAIL). By using 6-axis, (five revolute joints and one prismatic joint), this design permits a closed-form kinematics solution which could easily be calculated despite the limitations of the first computers. [1]. These robots are classified as serial robots since all the bodies and joints form a serial chain. Thirty years later, the majority of industrial robots still have a simple architecture derived from this robot [2]. Also, for many years, serial robots have been designed with two distinct units: 
the robotic arm to position the end-effector and then the wrist to orient the end-effector, meaning (i) three articulations for translation and (ii) three pivot joints with concurrent axes for rotation. We can then summarize their kinematics characteristics as (i) a trivial direct kinematic model, (ii) an inverse kinematic model with only eight easily identifiable solutions (elbow up, elbow down. shoulder left, shoulder right) and (iii) a regular workspace (spherical, cylindrical or cubic). The main problem with serial robots is their lack of rigidity which is solved by more mass resulting in less accelerations. If the parallel robots can solve this problem, they bring new challenges like (i) the complexity of their kinematic models, (ii) the presence of singularities inside their workspace and especially (iii) a low ratio between the workspace and the size of the robot [3]. Seeking a compromise led to the creation of (i) hybrid robots, and (ii) parallel robots with several actuators per kinematics chain (not fully parallel). The Tripteron is the parallel robot with three degrees of freedom having the simplest kinematic model which is as a simple Cartesian serial robot of the PPP type [4]. For spatial parallel robots, finding architectures with simple kinematic models remains an open problem. The purpose of this article is to use the 3-PPPS robot with a U-shaped base where the Euler angles are implemented for the orientation of the mobile plate-form [9].

The outline of this paper is as follows. First, we introduce the 3-PPPS parallel robot design parameters with simplifications which permit to study its joint space and workspace in a three dimensional space. In the next section, we will define the inverse and the direct kinematic models. In the later section, we study its singular configuration to provide the definition of an aspect included the "home" pose of the robot.

\section{Parameters and kinematics of the 3-PPPS parallel robot with U-shape base}

The results of the first work done at Monash University on the 3-PPPS introduced the MEPaM robot [5]. In the first design, the first actuators of the kinematics chains are located in orthogonal directions. For each leg, the three prismatic joints are placed orthogonally. With this design, it has been shown that the robot admits up to six solutions to the direct kinematic problem and it is able to follow non-singular assembly mode-changing trajectories. Another invariant is that the parallel singularity postures depend only on the orientation of the end-effector. Thus it is possible to study the workspace in a 3 dimensional space only. For the MEPaM manipulator, using a variable change, the joint space can also be studied in a 3 dimensional space. These properties allow us to calculate and display the domains without singularities of this robot. Another architecture was introduced where the two first actuated joints are located on the faces of a prism [5]. From the kinematic point of view, this design is simpler than the MEPaM, but the joint space needs to be analyzed in a five dimensional space. Recently a novel design was proposed where the actuated prismatic joints were located in a U-shaped base, as shown in Figure 1 [9]. To 


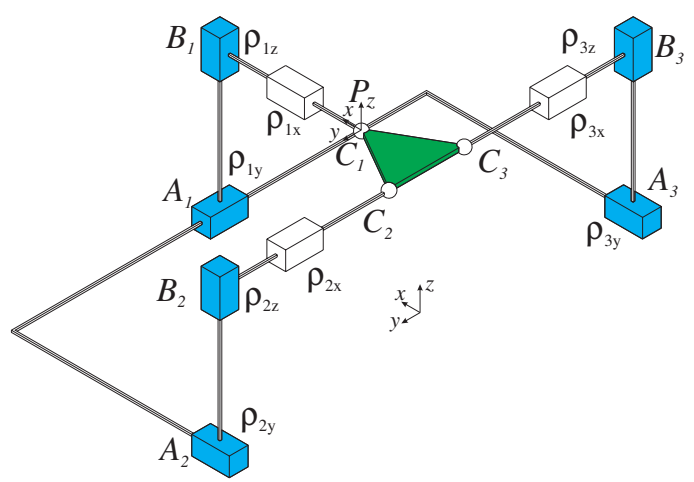

Fig. 1. The 3-PPPS parallel robot and its parameters in its "home" pose with the actuated prismatic joints in blue, the passive joints in white and the mobile platform in green

define the end-effector orientation, the $\mathrm{A}, \mathrm{B}$ and $\mathrm{C}$ angles define the rotation around the $\mathrm{X}, \mathrm{Y}$ and $\mathrm{Z}$ axes respectively [8]. We will use the following rotation matrix $\mathbf{R}=\mathbf{R}(x) \mathbf{R}(y) \mathbf{R}(z)$

$$
\mathbf{R}=\left[\begin{array}{ccc}
C_{\theta} C_{\sigma} & -C_{\theta} S_{\sigma} & S_{\theta} \\
S_{\phi} S_{\theta} C_{\sigma}+C_{\phi} S_{\sigma} & -S_{\phi} S_{\theta} S_{\sigma}+C_{\phi} C_{\sigma}-S_{\phi} C_{\theta} \\
-C_{\phi} S_{\theta} C_{\sigma}+S_{\phi} S_{\sigma} & C_{\phi} S_{\theta} S_{\sigma}+S_{\phi} C_{\sigma} & C_{\phi} C_{\theta}
\end{array}\right]
$$

where $C_{X}=\cos (X)$ and $S_{X}=\sin (X)$ for $X=\phi, \theta$ or $\sigma$ associated with the A, $\mathrm{B}$ and $\mathrm{C}$ angles respectively.

\subsection{Geometric parameters}

Similarly to [9], the three kinematics chains are identical and comprised of two actuated prismatic joints plus one passive prismatic joint and a spherical joint (Figure 1). The axes of the first three joints form an orthogonal reference frame. It may be noted that the placement order of the passive and active joints is not important. Thus, all the passive joints can be moved on the base of each leg to simplify the design. We have defined an origin $A_{i}$ for each leg as

$$
\mathbf{A}_{1}=[2,0,0]^{T}, \quad \mathbf{A}_{2}=[0,2,0]^{T}, \quad \mathbf{A}_{3}=[0,-2,0]^{T}
$$

The coordinates of the point $C_{1}$ are $\rho_{1 x}, \rho_{1 y}$ and $\rho_{1 z}$, where the last two are actuated. The coordinates of $C_{2}$ and $C_{3}$ are obtained by a rotation around the $z$ axis by $\pi / 2$ and $-\pi / 2$, respectively.

$$
\mathbf{C}_{1}=\left[\rho_{1 x}, \rho_{1 y}, \rho_{1 z}\right]^{T}, \quad \mathbf{C}_{2}=\left[-\rho_{2 y}, \rho_{2 x}, \rho_{2 z}\right]^{T}, \quad \mathbf{C}_{3}=\left[\rho_{3 y},-\rho_{3 x}, \rho_{3 z}\right]^{T}
$$

The origin on the mobile equilateral platform is similar to the parameters used in [9], meaning on the mobile platform.

$$
\mathbf{V}_{1}=[0,0,0]^{T}, \quad \mathbf{V}_{2}=[-\sqrt{3} / 2,1 / 2,0]^{T}, \quad \mathbf{V}_{3}=[-\sqrt{3} / 2,-1 / 2,0]^{T}
$$


Only the mobile platform orientation is positioned at the "home" posture to obtain $\phi, \theta, \sigma$ equal to zero. The moving platform coordinates, in the fixed reference frame, can be written as

$$
\mathbf{W}_{i}=\mathbf{R V}_{i}+\mathbf{P} \quad \text { where } \quad \mathbf{P}=\left[\begin{array}{ll}
x y & y
\end{array}\right]^{T}
$$

\subsection{Constraint equations}

From the nine equations coming from Eq. 5 and the position of points $C_{i}$, we can easily remove the passive joints, and thus obtain new constraint equations

$$
\begin{aligned}
\rho_{1 y}-y & =0 \\
\rho_{1 z}-z & =0 \\
C_{\theta} C_{\sigma} \sqrt{3}-C_{\theta} S_{\sigma}-2\left(\rho_{2 y}+x\right) & =0 \\
\sqrt{3}\left(S_{\phi} S_{\sigma}-C_{\phi} S_{\theta} C_{\sigma}\right)+C_{\phi} S_{\theta} S_{\sigma}+S_{\phi} C_{\sigma}+2\left(\rho_{2 z}-z\right) & =0 \\
C_{\theta} C_{\sigma} \sqrt{3}+C_{\theta} S_{\sigma}+2\left(\rho_{3 y}-x\right) & =0 \\
\sqrt{3}\left(S_{\phi} S_{\sigma}-C_{\phi} S_{\theta} C_{\sigma}\right)-C_{\phi} S_{\theta} S_{\sigma}-S_{\phi} C_{\sigma}+2\left(\rho_{3 z}-z\right) & =0
\end{aligned}
$$

We can notice that the symmetrical property of the mobile platform remains after the elimination of $\rho_{1 x}, \rho_{2 x}$ and $\rho_{3 x}$.

\subsection{Change of variables}

We introduce a change of variables to reduce the joint space dimension from six to three where three coordinates are equal to zero [9].

$$
\begin{array}{lll}
\mu_{1 x}=\rho_{1 x}-\rho_{2 y} & \mu_{1 y}=\rho_{1 y}-\rho_{1 y}=0 & \mu_{1 z}=\rho_{1 z}-\rho_{1 z}=0 \\
\mu_{2 x}=\rho_{2 x}-\rho_{1 y} & \mu_{2 y}=\rho_{2 y}-\rho_{2 y}=0 & \mu_{2 z}=\rho_{2 z}-\rho_{1 z} \\
\mu_{3 x}=\rho_{3 x}+\rho_{2 y} & \mu_{3 y}=\rho_{3 y}-\rho_{2 y} & \mu_{3 z}=\rho_{3 z}-\rho_{1 z}
\end{array}
$$

The remaining six parameters are three passive prismatic joints and three active prismatic joints. With this change of variables, the coordinates of the mobile platform are also translated but its orientation does not change.

$$
x^{\prime}=x-\rho_{2 y} \quad y^{\prime}=y-\rho_{1 y} \quad z^{\prime}=z-\rho_{1 z}
$$

This simplification is similar to the case where the three first prismatic joints are orthogonal as in [6]. If we apply the change of variable (12-14) in the constraint equations (6-11), we obtain the following

$$
\begin{aligned}
\left(\sqrt{3} C_{\sigma}-S_{\sigma}\right) C_{\theta}-2 x^{\prime} & =0 \\
\left(-\sqrt{3} C_{\phi} S_{\theta}+S_{\phi}\right) C_{\sigma}+\left(\sqrt{3} S_{\phi}+C_{\phi} S_{\theta}\right) S_{\sigma}+2 \mu_{2 z} & =0 \\
\left(\sqrt{3} C_{\sigma}+S_{\sigma}\right) C_{\theta}+2 \mu_{3 y}-2 x^{\prime} & =0 \\
\left(-\sqrt{3} C_{\phi} S_{\theta}-S_{\phi}\right) C_{\sigma}+\left(\sqrt{3} S_{\phi}-C_{\phi} S_{\theta}\right) S_{\sigma}+2 \mu_{3 z} & =0
\end{aligned}
$$




\section{Inverse and direct kinematics of the 3-PPPS parallel robot}

Finding the solutions of the direct geometric model of a parallel robot is often a difficult problem, especially for robots with six degrees of freedom. If in [9] for the mechanism studied, a compact form was found, the use of Euler angles could lead to more complex expressions. With the Siropa library, it is possible to obtain the kinematic models by using the Gröbner based elimination method by changing the constraint equation in an algebraic form. A classical approach can be used to transform the trigonometric equations to algebraic ones as in [10].

\subsection{Inverse kinematic model}

Thanks to the choice of the origin on the mobile platform which is fixed at $C_{1}$, it is straightforward to find the joint positions of the first kinematics chain :

$$
\rho_{1 y}=y \quad \text { and } \quad \rho_{1 z}=z
$$

For the two other kinematics chain, the result keeps the symmetric property and becomes

$$
\begin{aligned}
\rho_{2 y} & =\frac{C_{\theta}\left(C_{\sigma} \sqrt{3}-S_{\sigma}\right)}{2}-x \\
\rho_{2 z} & =\frac{\sqrt{3}\left(C_{\phi} S_{\theta} C_{\sigma}-S_{\phi} S_{\sigma}\right)-C_{\phi} S_{\theta} S_{\sigma}-S_{\phi} C_{\sigma}}{2}+z \\
\rho_{3 y} & =-\frac{C_{\theta}\left(C_{\sigma} \sqrt{3}+S_{\sigma}\right)}{2}+x \\
\rho_{3 z} & =\frac{\sqrt{3}\left(C_{\phi} S_{\theta} C_{\sigma}-S_{\phi} S_{\sigma}\right)+C_{\phi} S_{\theta} S_{\sigma}+S_{\phi} C_{\sigma}}{2}+z
\end{aligned}
$$

The computational cost to solve the inverse kinematics handles six trigonometric functions (sine and cosine), the square of three, 14 multiplications/divisions and 11 additions/subtractions if we used the code generation of Maple with the optimization function to create intermediary variables.

\subsection{Direct kinematic model}

The computation of the position does not depend on the choice of parameters for the orientation. For the position, we have the similar results than in [9].

$$
\begin{aligned}
x & =\frac{\mu_{2 z}{ }^{2} \mu_{3 y}-\mu_{2 z} \mu_{3 y} \mu_{3 z}-\mu_{3 y} / 2}{\left(\mu_{2 z}-\mu_{3 z}+1\right)\left(\mu_{2 z}-\mu_{3 z}-1\right)} \\
& \pm \frac{\sqrt{\left(\mu_{2 z}{ }^{2}-\mu_{2 z} \mu_{3 z}+\mu_{3 z}{ }^{2}-3 / 4\right)\left(\mu_{2 z}{ }^{2}-2 \mu_{2 z} \mu_{3 z}+\mu_{3 y}{ }^{2}+\mu_{3 z}{ }^{2}-1\right)}}{\left(\mu_{2 z}-\mu_{3 z}+1\right)\left(\mu_{2 z}-\mu_{3 z}-1\right)}
\end{aligned}
$$


For the orientation, for any values of $x$, we obtain

$$
\begin{gathered}
\theta_{1}= \pm \arccos \left(2 \sqrt{\frac{\mu_{3 y}^{2}-\mu_{3 y} x+x^{2}}{3}}\right) \quad \text { and } \quad \theta_{2}= \pm\left(\pi-\theta_{1}\right) \\
\sigma_{1}= \pm \arccos \left(\frac{\mu_{3 y}-2 x}{2 \sqrt{\mu_{3 y}^{2}-\mu_{3 y} x+x^{2}}}\right) \quad \text { and } \quad \sigma_{2}= \pm\left(\pi-\sigma_{1}\right) \\
\phi_{1}= \pm \arccos \left(\sqrt{\frac{3 / 4-\rho_{2 z}^{2}+\rho_{2 z} \rho_{3 z}-\rho_{3 z}^{2}}{\rho_{3 y}^{2}-\rho_{3 y} x+x^{2}}}\right) \quad \phi_{2}= \pm\left(\pi-\phi_{1}\right)
\end{gathered}
$$

The computational cost to evaluate one solution comprises three arccos functions, three square roots, 17 multiplications, 8 divisions, 8 additions, and 12 subtractions. In [9], it was shown that there exists four direct kinematic solutions for any joint values. We have a similar result because two sets of $\theta$ can define the same mobile platform orientation. So, we keep that a value for $\theta$, such that $\pi / 2 \leq \theta \leq \pi$. We have 16 solutions considering all $\theta, \sigma$ and $\phi$ permutations but only four are solution of the constraint equations.

\section{Workspace and singular configurations of the 3-PPPS parallel robot}

From the constraint equations, we can easily determine the inverse Jacobian of the robot and its parallel singularities

$$
C_{\phi} C_{\theta}^{2}\left(S_{\phi} S_{\theta} S_{\sigma}-C_{\phi} C_{\sigma}\right)=0
$$

As Eq. 29 is factored, we can isolate three surfaces as is shown in Fig.2. The first term is represented by the blue surface, the second term by the green surface and the last one by the red surface.

We can also notice that the coordinate change does not change the singularity locus in the joint space, [9]. However, the computational cost is higher because in the Gröbner based elimination, we have to remove six more parameters that are required with quaternions. Using the Cylindrical Algebraic Decomposition, the aspect is characterized including the robot "home" position, [7]. This is done by only two cells. The orientation workspace has the following distinct limitations which can be directly related to the platform rotations, figure 3 :

- Along the $\theta$ and $\phi$ angles, the complete range of motion is from $-\pi / 2$ until $\pi / 2$;

- Along the $\phi$ angle, the complete motion ranges from $-\pi$ until $\pi$ intersecting a plane at $\phi=\pi / 2$, if $\alpha>0$ then $-\pi / 2 \leq \theta \leq 0$ the this plane segment looks like a rectangle; 


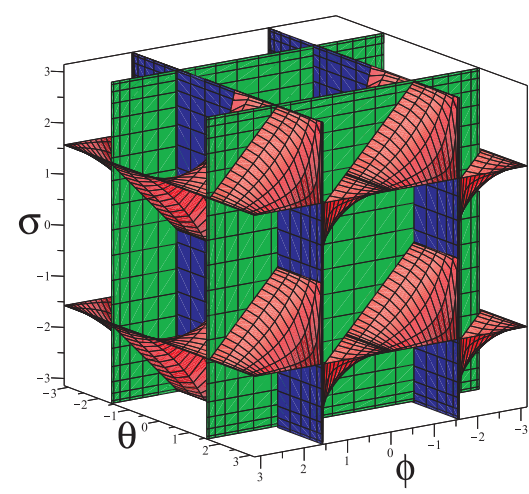

Fig. 2. The three singularity surface of the Fig. 3. The aspect of the 3-PPPS parallel 3-PPPS parallel robot

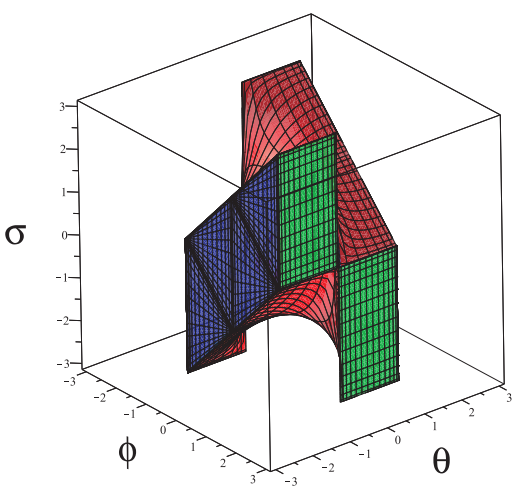

robot around the "home" pose

- On the other side, accordingly, intersecting a plane at $\phi=-\pi / 2$, if $\alpha<0$ then $0 \leq \theta \leq p i / 2$ meaning a symmetrical rectangle appears. These two symmetrical rectangles will evolve towards the other side as $\phi$ is changed from $\pi / 2$ to $-\pi / 2$. In the middle, intersecting a plane at $\phi=0$, the workspace is a square with sides at $-\pi / 2$ and $\pi / 2$.

Hence, the $\theta$ range divides itself differently if $\alpha$ is positive or negative with traveling symmetrical rectangles. This results from the selected symmetries and axis positions. These traveling or sliding rectangles actually produce two interesting saddle points on the workspace limit where $\alpha=0$ and $\theta=0$. Hence, the graph solution is geometrically confirming the displacement limitations that can be intuitively understood from manipulator observation. These workspace distinct limits come from the passive prismatic joints.

\section{Conclusions}

In this article, we introduced kinematics modeling where the orientation representation of the 3-PPPS mobile platform implemented the Euler angles as it is often represented on many machine tools followed by a significant variable change. We can see that the equations of the direct and inverse kinematics model remain very compact. An estimate of the calculation cost is presented. Since the equations are quadratic, it is possible to obtain an analytical expression which is not possible for most parallel robots with 6 degrees of freedom. A representation of the aspect encompassing the "home" position is given. Conversely to the 5 -axis Orthoglide [11] or 4-axis Kanuk [12], the 3-PPPS parallel robot is using 6 identical linear or prismatic actuators as a Gough-Stewart platform. Inside each kinematic chain, the passive and active joint order can be changed to obtain, for example, the passive joint fixed into the base without altering the singularity 
and aspect results. Future works will be done to characterize the stiffness model as well as to know the influence of the joint limits in the spherical joints.

\section{References}

1. Paul, Richard P, Robot manipulators: mathematics, programming, and control: the computer control of robot manipulators, Richar Paul, 1981.

2. Bruno, Siciliano and Oussama, Khatib. 2007. Springer Handbook of Robotics. Springer-Verlag New York, Inc., Secaucus, NJ, USA.

3. Merlet, J. P., "Parallel robots", Springer Science \& Business Media, 2006.

4. Kong, X. and Gosselin, C.M., 2002, "Type synthesis of linear translational parallel manipulators," in Lenarčič, J. and Thomas, F. (editors), Advances in Robot Kinematic, Kluwer Academic Publishers, June, pp. 453-462.

5. Chen, C., Gayral, T., Caro, S., Chablat, D., Moroz, G., "A Six-Dof Epicyclic-Parallel Manipulator," Journal of Mechanisms and Robotics, American Society of Mechanical Engineers, Vol. 4 (4), pp.041011-1-8, 2012.

6. Caro, S., Wenger, P. and Chablat, D., "Non-Singular Assembly Mode Changing Trajectories of a 6-DOF Parallel Robot," ASME Design Engineering Technical Conferences \& Computers and Information in Engineering Conference IDETC/CIE, Chicago, August 12-15, USA, 2012.

7. Manubens M., Moroz G., Chablat D., Wenger P., Rouillier F., Cusp Points in the Parameter Space of Degenerate 3-RPR Planar Parallel Manipulators, ASME Journal of Mechanisms and Robotics, Vol. 10(1), 2012.

8. Lamikiz A. , López de Lacalle L. N. , Ocerin O., Díez D., and Maidagan E., "The Denavit and Hartenberg approach applied to evaluate the consequences in the tool tip position of geometrical errors in five-axis milling centres," Int J Adv Manuf Technol (2008) 37:122139.

9. Chablat D., Baron L., Jha R., "Kinematics and Workspace Analysis of a 3PPPS Parallel Robot with U-Shaped Base," International Design Engineering Technical Conferences \& Computers and Information in Engineering Conference, 2017.

10. Moroz G., Rouiller F., Chablat D., Wenger P., On the determination of cusp points of 3-RPR parallel manipulators, Mechanism and Machine Theory, Vol. 45(11), pp. 1555-1567, 2010.

11. Caro S., Chablat D., Lemoine P., Wenger P., Kinematic Analysis and Trajectory Planning of the Orthoglide 5-Axis, Proceedings of the ASME 2015 , International Design Engineering Technical Conferences \& Computers and Information in Engineering Conference, Aug 2015, Boston, United States, 2015.

12. Rolland L. The Manta and the Kanuk: Novel 4-DOF Parallel Mechanisms for Industrial Handling. In proceedings of the ASME, Dynamic Systems and Control Division - 1999, vol 67, pp 831 - 844, Conference IMECE'99, Nashville, novembre 14-19, 1999. 